\section{NCy 07}

INFLUENCE OF MESNA ON THE TOXICWY AND THERAPEUTIC EFFICACY OF CISPLATIN T. Wagner and G. Bohlmann

Sulfur compounds such as sodium thiosulfate are known to reduce the toxicity of cisplatin (Howe11,S.B. et a1., Cancer Res.43,1426,1983). Another thiol mesna (sodium-2-mercaptoethansulfonate) efficiently acts against the urotoxicity of activated cyclophosphamide and its coadministration in cyclophosphamide therapy has become clinical standard by now. Since cyclophosphamide is often combined with cisplatin for cancer treatment the influence of mesna on the acute toxicity and therapeutic efficacy of cisplatin against $\mathrm{S} 180$ ascites sarcoma was investigated by us in NMRI mice. After separate $i . p$. injections of cisplatin and mesna ( $800 \mathrm{mg} / \mathrm{kg}$, one minute later) an increase of the LD50 from $17,0 \mathrm{mg} / \mathrm{kg}$ to $25,5 \mathrm{mg} / \mathrm{kg}$ was found. Simultaneously the CD50 value of cisplatin in treatment of $\mathrm{S} 180$ ascites sarcoma increased from $1,3 \mathrm{mg} / \mathrm{kg}$ to $4,0 \mathrm{mg} / \mathrm{kg}$. Thus the concurrent injection of mesna reduced both the toxicity and - even to a greater extent - the antitunour effect of cisplatin. Therefore, despite the possible application of a higher dose of cisplatin the therapeutic index (LD50/CD50) decreased from 11,3 to 6,3 in the presence of mesna. Application of mesna i.p. 1 hour before or after cisplatin was ineffective. Also i.v. application of mesna had no influence on the toxicity (LD50 $15,5 \mathrm{mg} / \mathrm{kg}$ ) and therapeutic efficacy (CD50 1,5 mg/ kg) of $i . p$. cisplatin in sl80 ascites sarcoma. On the contrary, i.p. as well as i.v. applicated sodium thiosulfate was 3 -times as effective against lethal toxicity of $1 . p$. cisplatin when compared with equimolar doses of i.p. mesna. Following our results, only the sirnltaneous application of cisplatin and mesna at the same route of injection should be avolded to present a loss of antitumour efficacy in cancer chemotherapy. Otherwise there will be no distinct interference.

Klinik für Innere Medizin der Medizinischen Universität zu Lübeck, Ratzeburger Allee 160, D-2400 Lübeck.

\section{NCy 08}

CHEMOSENSITIZATION BY CYCLOSPORINE A, A NEW SIRATEGY IN DRUG DEVELOPMENT

R. Osieka, kloke 0., Seeber, 5, scheulen, M.,
Pannenbäcker, R. (a.G.\}, SoII, D. (a.G.), Glatte, P. \{a.G\} and Schmiat $C . G$.

Apart from their increasing role in cardiovascular medicine, calcium channel blockers fulfil the criteria of true chemosensitization. The enhancement is dose-dependent and varies with the antineoplastic agent in use. Furthermore. the degree of chemosensitization correlates inversely with the inherent chemosensitivity of a cell. This makes chemosensitization an attractive approach to overcome drug resistance in tumor cells. Unfortunately, acute cardiotoxicity does not allow for plasma levels of established calcium channel blockers, e.g. verapamil, sufficiently high to induce such chemosensitization in patients.

Therefore, a drug with iess acute taxicity was desirable for exploitation of this new treatment strategy. We here describe the interaction of cyclasporine $A$, hitherta used exclusively for immunosuppression, with several antineoplastic agents and ionizing irradiation. Using 11210 cells, cyclosporine $A$ at a concentration of 2 $\mu \mathrm{g} / \mathrm{ml}$ markedly enhanced the cytotoxicity of

etoposide and adriamycin but not of cisplatin, melphalan or ionizing irradiation. The interaction with etoposide is partialiy mediated by increased infracellular drug retention as shown by uptake studies with ${ }_{\text {H-etoposide }}$ and determination of DNA-damage in the presence or absence of cyclosporine $A$. In animal experiments and in patients bluod levels in excess of $2 \mu \mathrm{g} / \mathrm{ml}$ cyclosparine

A have been achieved without acute toxicity. Using a human embryonal carcinoma xenograft, there was a cleax growth delay induced by the combined use of etoposide and cyclosporine $A$, but no effect of etoposide alone. Extensive in vitro studies with peripheral blood cells from normal donors of leukemic patients showed that this effect is present both in normal and neoplastic cells. There was a trend to greater enhancement in drug resistant cells. Westdeutsches Tumorzentrum, HufelandstraBe 55, 43 Essen 1

\section{NCy 09}

METABOLISM OF ETHER LIPIDS IN LEUKAEMIC CELLS E.A.M. Fleer, D. -J. Kim, C. Unger and H. Eibl

Ajm of research: (Alkyl)-lysophospholipids have been known to exert strong antineoplastic activity. However, a comparison between (alkyl)-lysophospholipids of different chemical structures revealed that differences in the cytotoxic effects to tumour cells cannot be explained by the surface active properties of the compounds as shown by haemolysis experiments on human erythrocytes. This experimental observation indicates that metabolism of the compounds is involved. We used radiolabelled (alkyl)-lysophospholipids to understand the metabolic fate of these compounds and possibly the mode of cytotoxic action.

Methods: Synthesis of 1-octadecy1-2-0-methyl-rac-glycero3-phospho (3imethyl) choline has been described (H. Eibl, Angew. Chem. 23, 257, 1984). Raji cells were incubated with the radiolabelled (alkyl)-lysophospholipid and were analyzed for metabolic products after 24,48 and 72 hours with thin-layer chromatography.

Results: The only radiolabelled product formed during the incubation is diacyl-sn-glycero-3-phosphocholine (lecithine). The formation of lecithine is linear with time and strongly parallels cell. death. The enzyme reaction described above is the first experimental observation of a direct transfer of the intact phosphocholine group from (alkyl)-lysophosphocholine to diacylglycerol.

Conclusions: The biosynthesis of lecithine cannot result in cell death. We, therefore, conclude that the other product of the metabolic conversion, 1-octadecyl-2-o-methy1rac-glycerol, is the real cell toxine, because cell death is always accompanied by the formation of this product. This is the first study which shows that not the actual (alkyl)-lysophospholipids are the toxic principle but the alkyl glycerols formed by the action of phospholipase Clike enzymes (prodrug principle).

Max-Planck-Institut für biophysikalische Chemie, Am Fabberg, D-3400 Göttingen

\section{NCy 10}

(ETHER)-PHOSPHOLIPIDS IN LIPOSOMES: THE INFLUENCE OF SURFACE CHARGE ON ORGAN DISTRIBUPTION

\section{P. Kaufmann-Kolle, C. Unger and $H$. Eibl}

Aim of research: The preparation of liposomes from phospholipids in water dispersion generates unique carrier molecules, which can be used for the transport of lipophilic and hydrophilic cytostatics. It was the aim of this study to look for modifications (chemical structure, surface charge) which result in organ specificity.

Methods: Liposomes were prepared with the French Press using well-defined synthetic phospholipids. The stability of the liposomes was tested by inclusion of carboxyfluorescein. To analyze the biodistribution of liposomes, (3i)inulin was included, and the radioactivity found in the organs was determined with a tissue oxygenizer.

Results: In a detailed analysis we can show that biodistribution of liposomes depends not only on the net charge but also on the chemical structure of the molecules. The very new findings are that some negative-charged liposomes remain in the blood circulation for longer time periods. Using a new positive-charged (ether)-phospholipid we observe a dramatic effect. These liposomes are eliminated from the blood within a few minutes after i.v. injection and accumulate almost exclusively in the Iiver. This behaviour differs strongly from the results with stearylamine (R.L. Juliano et al., B.B. Res. Commun. 63, 651, 1975). Conclusions: The results suggest that positive-charged liposomes have excellent target properties to the liver and should be useful in new therapeutic strategies. The Iiposomes can be used as carrier for water solubie and lipophilic drugs. Thus, this special kind of positive-charged liposomes may be useful in treating liver tumours or liver metastases by simple $i . v$. injections (organ perfusion by i.v. injection).

Max-Planck-Institut für biophysikalische Chemie, Am Faßberg, D-3400 Göttingen 